\title{
Preoperative evaluation of persistent cloaca using contrast-enhanced ultrasound in an infant
}

\author{
Xue-Yun Lin', Xiao-Hong Xu¹, Yu-Ping Yang ${ }^{1}$, Jiang Wu², Xiao-Hui Xian², Xiao-Dong Chen ${ }^{3}$ \\ ${ }^{1}$ Department of Ultrasound, ${ }^{2}$ Department of Pediatric Surgery, ${ }^{3}$ Department of Radiology, Affiliated Hospital of \\ Guangdong Medical University, Guangdong, China
}

\begin{abstract}
Persistent cloaca (PC) is the most intricate anorectal malformation. Contrast-enhanced ultrasound (CEUS) is safe for hepatic, renal, splenic, vascular, and intracavitary assessment in children, but it is little applied for cloacal malformation. Our results demonstrate that CEUS can not only display the uterine, vagina, bladder, urethra, rectum, and rectovaginal fistula, but also measure the length of the common channel, bladder neck to the common channel, and distal urethra to the perineum. To our knowledge this may be the first report of preoperative evaluation by CEUS in an infant with PC.
\end{abstract}

Keywords: persistent cloaca; anorectal malformation; contrast-enhanced ultrasound

\section{Introduction}

Persistent cloaca (PC) is the most intricate anorectal malformation characterized by a convergence of the urethra, vagina, and rectum forming into a narrow common channel in the perineum $[1,2]$. Antenatal ultrasound and fetal magnetic resonance imaging (MRI) can provide the important clues for prenatal diagnosis of cloacal malformations [3]. Preoperative visualization and measurement of structural relationships is obligatory for the surgical

Received 21.05.2019 Accepted 28.07.2019

Med Ultrason

2020, Vol. 22, No 2, 250-252

Corresponding author: Xiao-Hong Xu

Department of Ultrasound, Affiliated Hospital

of Guangdong Medical University

57 Southern Renmin Avenue, Xiashan District,

Zhanjiang, Guangdong, 524001, China

E-mail: 13828297586@139.com

Phone: 86-759-2387531

Fax: 86-759-2231754 repair, and preoperative evaluation methods including endoscopy, ultrasonography, fluoroscopy contrast study (FCS), 3-D fluoroscopic reconstruction, and MRI have been feasible $[1,2,4]$. Contrast-enhanced ultrasound (CEUS) has been used safely for hepatic, renal, splenic, vascular, and intracavitary assessment in children $[5,6]$. However, CEUS is little applied for cloacal malformation even though it may become the promising approach [7]. We describe here the preoperative evaluation of PC using CEUS in an infant.

\section{Case report}

A female infant weighing $2800 \mathrm{~g}$ was vaginally born at 40 weeks and presented with a single perineal introitus. She was diagnosed with an anorectal malformation and underwent a transverse colostomy 1 month later. The patient was readmitted to our hospital for complete perineum reconstruction at the age of 6 months. Physical examination revealed a single perineal orifice 


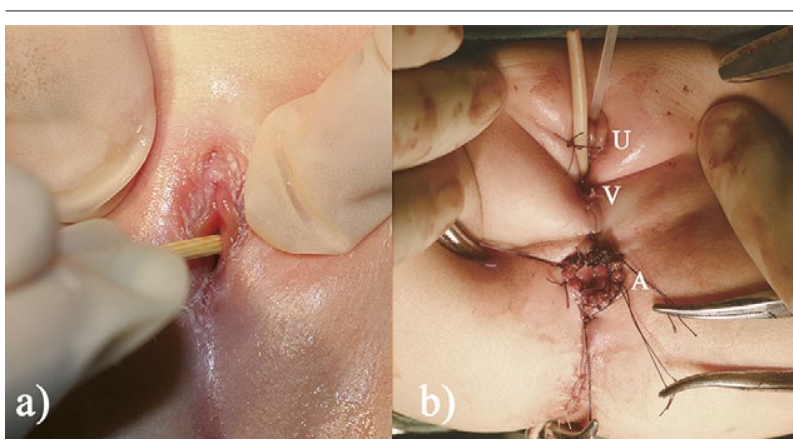

Fig 1. Perioperative photographs of a 6-month-old female infant: (a) a single perineal opening; (b) the reconstructed urethra (U), vagina (V) and anus (A).

(fig 1a). Cardiac and abdominal ultrasound results were unremarkable. Pelvic ultrasound displayed a $1.6 \times 0.8 \mathrm{~cm}$ well-defined uterus with a uterus/cervix ratio of 2:1 (fig 2a).

Abdomen MRI was performed using 3.0-T magnet and adult abdomen coil without sedation. The slice thickness was $5 \mathrm{~mm}$. MRI showed a $5.9 \times 4.8 \times 2.1 \mathrm{~cm}$ bladder and $2.0 \times 1.0 \times 0.7 \mathrm{~cm}$ uterine-like structure. The distal urethra, vagina, distal rectum, rectovaginal fistula, and common channel were not clear-cut (fig 3).

CEUS was performed without anesthesia. A 6F Foley catheter was placed into the rectum through the perineal opening, and instilled with sulfur hexafluoride microbubbles (Sonovir). The catheter was slowly withdrawn with the injection of the contrast agent. The rectum, rectovaginal fistula and vagina were simultaneously displayed at a distance of $1.6 \mathrm{~cm}$ from the perineal opening, while the urethra and bladder were seen at $1.1 \mathrm{~cm}$. The length of the common channel (distance from the distal vagina to perineum), bladder neck to common channel, and distal urethra to perineum was $1.6 \mathrm{~cm}, 1.5 \mathrm{~cm}$, and $1.1 \mathrm{~cm}$, respectively (fig $2 \mathrm{~b}-\mathrm{d}$ ). These results support the diagnosis of PC.

The patient underwent a posterior sagittal anorectoplasty (PSARP) and vaginal posterior wall mobilisation (fig 1b).

\section{Discussions}

The common canal (CC) refers to the canal where the urethra, vagina, and rectum fistula converge into the perineum, and its length is the main determinant for operative programs. The $\mathrm{CC}$ length can be measured from the distal vagina or rectum fistula to the perineum (labia minora) [1]. PC is traditionally categorized into short $(<3 \mathrm{~cm})$ and long $(>3 \mathrm{~cm})$ types based on the length of the $\mathrm{CC}[1,2]$.

To date there is no gold standard method for preoperative assessment of PC. Endoscopy is hard to discern the spatial relationships between the structures [4]. MRI and FCS are the most common methods for preoperative assessment of PC, but they are difficult to precisely measure the length of CC. Traditional 2-D FCS images may present with structural overlap, image foreshortening, internal reference absence, and magnification errors, while MRI cannot clearly display the thin tubular structures such as urethra, rectal fistula and CC [1,8]. 3-D reconstruction with rotational fluoroscopy $[2,8]$ or Dyna-CT [4] has been successfully utilized for preoperative anatomical evaluation of complex cloacal malformations, but its disadvantages include general anesthesia and high radiation exposure. CT should be reserved for complex cases if MRI is unavailable [7]. 3-D MRI provides superior anatomical information for cloacal malformations [9], but real-time visualization or dynamic anatomic information is not obtained [8].

In this case, since the non-enhanced MRI did not determine the CC and confluence, CEUS was tried out. CEUS can not only display the uterine, vagina, bladder, urethra, rectum, and rectovaginal fistula, but also measure the length of CC, bladder neck to the common channel, and distal urethra to the perineum. Although PC is commonly accompanied by multiple systemic anomalies [1], our case showed no concomitant anomalies using conventional ultrasound, CEUS and MRI. PC was successfully corrected by PSARP and vaginal posterior wall mobilisation because of short PC $(\mathrm{CC}=1.6 \mathrm{~cm})$. These results imply that CEUS can be useful for the diagnosis

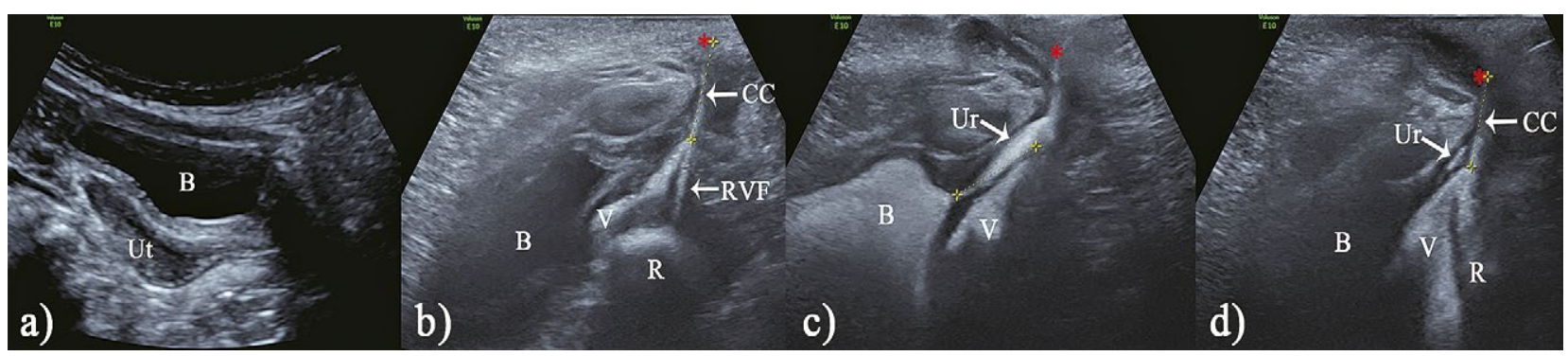

Fig 2. Ultrasound images: (a) pelvic ultrasound showed the uterus (Ut) and bladder (B); (b-d) contrast-enhanced ultrasound revealed vagina $(\mathrm{V})$, bladder $(\mathrm{B})$, urethra $(\mathrm{Ur})$, rectum $(\mathrm{R})$, rectovaginal fistula $(\mathrm{RVF})$, and common channel $(\mathrm{CC})$. 


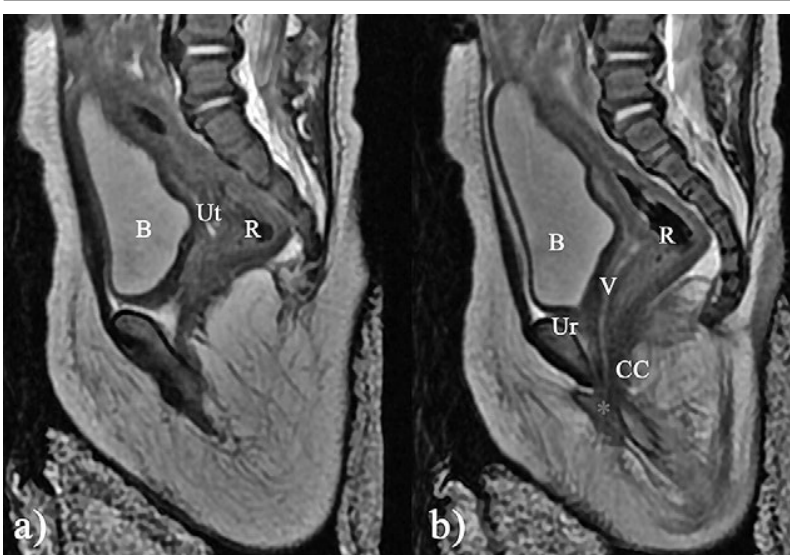

Fig 3. Sagittal MRI of the lower abdomen showed the rectum $(\mathrm{R})$, vagina $(\mathrm{V})$, uterus (Ut), bladder (B), urethra (Ur), and illdefined common channel (CC).

and preoperative visualization of PC. In addition, CEUS lacks ionizing radiation and can be performed without general anesthesia or sedation.

In conclusion, to our knowledge this may be the first report of preoperative evaluation by CEUS in an infant with PC. CEUS may be simple, effective, safe and inexpensive for the diagnosis and preoperative assessment of PC, which deserves to be further studied in the future.

\section{References}

1. Wang Z, Wu H, Wang Y, et al. Measuring the common canal of a persistent cloaca: can MRI replace conventional imaging? Clin Radiol 2019;74:488.e9-488.e15.
2. Reck-Burneo CA, Lane V, Bates DG, et al. The use of rotational fluoroscopy and 3-D reconstruction in the diagnosis and surgical planning for complex cloacal malformations. J Pediatr Surg 2019 Apr 4. doi: 10.1016/j.jpedsurg.2019.03.016.

3. Peiro JL, Scorletti F, Sbragia L. Prenatal diagnosis of cloacal malformation. Semin Pediatr Surg 2016;25:71-75.

4. Ahn JJ, Shnorhavorian M, Amies Oelschlager AE, et al. Use of $3 \mathrm{D}$ reconstruction cloacagrams and $3 \mathrm{D}$ printing in cloacal malformations. J Pediatr Urol 2017;13:395.e1-395. e6.

5. Torres A, Koskinen SK, Gjertsen H, Fischler B. Contrastenhanced ultrasound using sulfur hexafluoride is safe in the pediatric setting. Acta Radiol 2017;58:1395-1399.

6. Yusuf GT, Sellars ME, Deganello A, Cosgrove DO, Sidhu PS. Retrospective analysis of the safety and cost implications of pediatric contrast-enhanced ultrasound at a single center. AJR Am J Roentgenol 2017;208:446-452.

7. Riccabona M, Lobo ML, Ording-Muller LS, et al. European Society of Paediatric Radiology abdominal imaging task force recommendations in paediatric uroradiology, part IX: Imaging in anorectal and cloacal malformation, imaging in childhood ovarian torsion, and efforts in standardising paediatric uroradiology terminology. Pediatr Radiol 2017;47:1369-1380.

8. Patel MN. Use of rotational fluoroscopy and 3-D reconstruction for pre-operative imaging of complex cloacal malformations. Semin Pediatr Surg 2016;25:96101 .

9. Baughman SM, Richardson RR, Podberesky DJ, Dalrymple NC, Yerkes EB. 3-Dimensional magnetic resonance genitography: a different look at cloacal malformations. J Urol 2007;178:1675-1678. 